\title{
CONTENTS
}

1. AIM AND SCOPE OF THE JOURNAL.........................................................................................

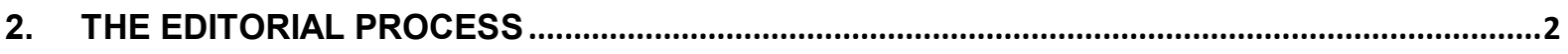

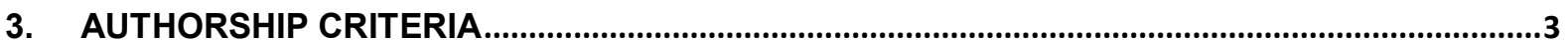

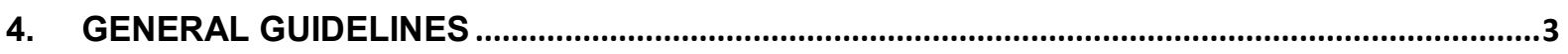

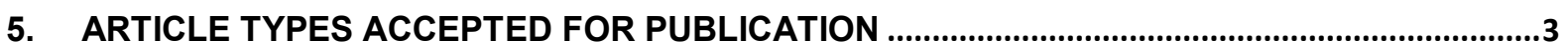

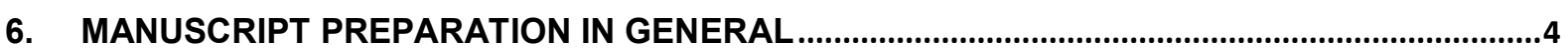

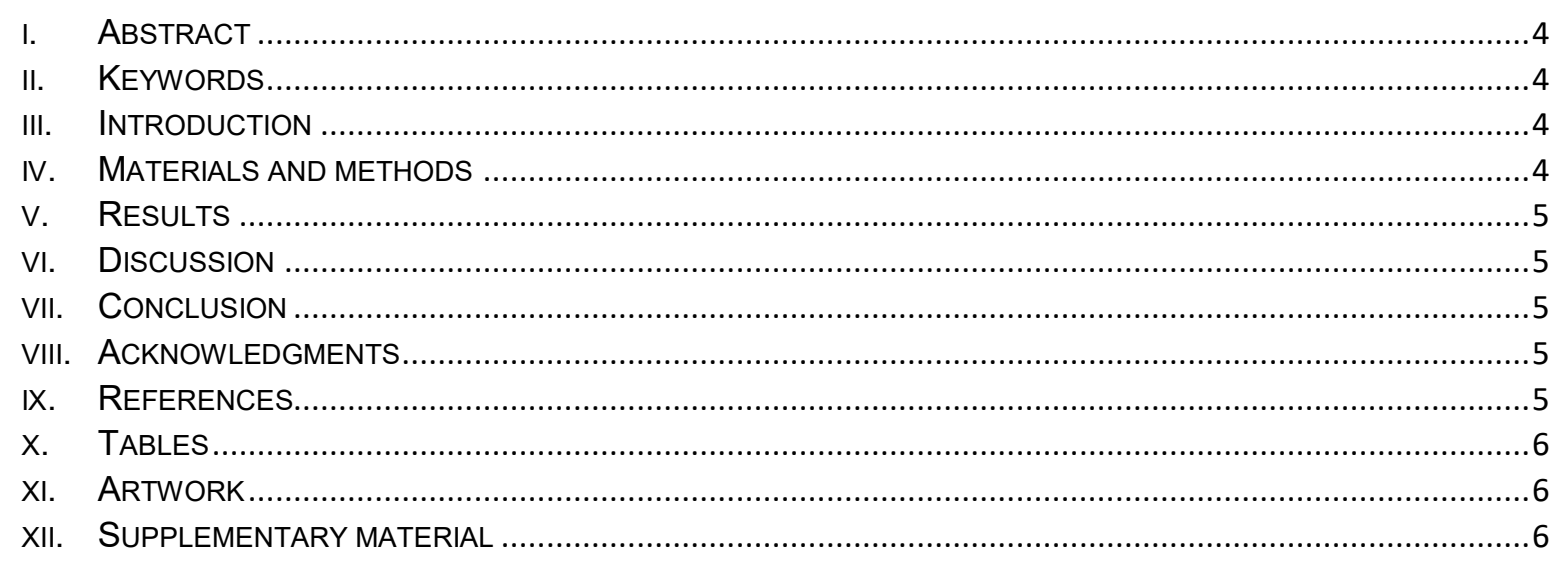

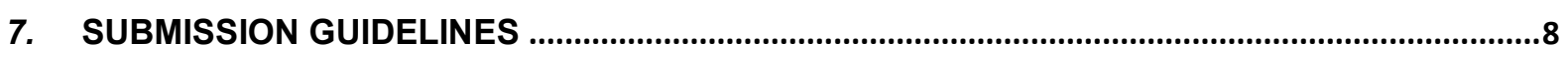


1. Aim and Scope of the Journal

Journal of Pharmaceutical Research (JPR) is a peer-reviewed, Open Access journal that publishes original research articles as well as review articles in the area of pharmaceutical science related subjects. The Journal publishes original research papers on both scientific and technical progress, and future trends. Contributions in the form of research articles, short communications, notes, case reports and reviews are considered for publication. The editors welcome original contributions that have not been and are not to be published elsewhere. The topics of the Journal include headache and its associated subjects.

\section{The Editorial Process}

A manuscript will be reviewed for possible publication with the understanding that it is being submitted to JPR alone at that point in time and has not been published anywhere, simultaneously submitted, or already accepted for publication elsewhere. All manuscripts received are duly acknowledged.

On submission, editors review all submitted manuscripts. Manuscripts with insufficient originality, serious scientific or technical flaws, or lack of a significant message are rejected before proceeding for formal peer-review.

Manuscripts that are found suitable for publication in JPR are sent to two or more expert reviewers. The journal follows a double-blind review process, where in the reviewers and authors are unaware of each other's identity.

Every manuscript is also assigned to a member of the editorial team, who based on the comments from the reviewers takes a final decision on the manuscript. The comments and suggestions (acceptance/rejection/revision in manuscript) received from reviewers are conveyed to the corresponding author. If required, the author is requested to provide a point by point response to reviewers' comments and submit a revised version of the manuscript.

Manuscripts accepted for publication are copy edited for grammar, punctuation, print style, and format. Page proofs are sent to the corresponding author.

The corresponding author is expected to return the corrected proofs within two days. The whole process of submission of the manuscript to final decision; sending and receiving proofs is completed online, with an average of 6 weeks duration.

As the journal is an open access one, publishing charges apply to the author on acceptance of the article.

Correspondence with the authors by any members of editorial team except Editor-inChief and Editor is strictly prohibited. 


\section{Authorship Criteria}

Authorship credit should be based only on substantial contributions to each of the three components mentioned below:

i. Concept and design of study or acquisition of data or analysis and interpretation of data;

ii. Drafting the article or revising it critically for important intellectual content; and

iii. Final approval of the version to be published.

Every manuscript should be accompanied by a contributor's form which describes about the contribution of each author towards the manuscript, with respect to the above authorship criteria. The originality of the manuscript has to be declared by the authors.

4. General Guidelines

i. Use only New Times Roman font.

ii. Prepare the manuscript in Microsoft Office Word with A4 size paper setting.

iii. 18 font for the title of the article.

iv. $\quad 14$ font for the headings which should be bold.

v. 12 font for sub-headings which should be bold.

vi. 12 font for the text.

vii. Do not use capital letters for title and sub-headings.

viii. Double-spacing should be maintained throughout the manuscript.

ix. Should number all of the pages of the manuscript consecutively, beginning with the title page.

\section{Article Types accepted for publication}

A manuscript with a research study relevant to the scope of the journal is expected from the authors. The study should be substantiated with appropriate study methodology, results, discussion and conclusion. Following are the types of manuscripts accepted for publication in the journal: Research article, Review article, Mini-review, Editorial, Short communication, Commentaries, Short notes. All the submitted manuscripts should follow the standard guidelines based on the types of manuscript, more details can be found in Table 1 . 
6. Manuscript Preparation in General

i. Abstract

The abstract should have the following subheadings: Objectives, Methods, Findings, Novelty and Keywords. The scientific terms appearing here for the first time should not be abbreviated. The word limit for the abstract is up to 250 words.

Please refer to the abstract word count in Table 2.

ii. Keywords

At the least 3 keywords and a maximum of 6 keywords can be included. They must be separated by commas to distinguish them. The first letter of all the keywords should be in capital letters

iii. Introduction

The introduction section should provide a context for your manuscript in brief. The introduction can include comprehensive information about the background, prior research in the field of study and objectives of research in the present study.

iv. Materials and methods

The materials and methods as the name suggests should detail the different materials and methods used in the study with relevant citations of previous studies. In this section, the scientific terms should be in the expanded form at their first appearance, they can be abbreviated in their subsequent appearances in the text.

If the manuscript includes trials on patients, the use of data of patients, confirmation about the consent from participant patients should be included. Information regarding the approval from the Institutional ethics committee should be part of this section as well. The reference to the methods should be cited with proper references for the readers to analyse and verify the reported results of the study.

This section should include all the experimental protocols gathered for the study for better understanding. The major methodology can be presented in the main manuscript, additional information about the methodology can be included as supplementary data.

The author is expected to maintain high stands of scientific integrity during the scripting of the manuscript. Recommendations as laid out by Committee on Publication Ethics (COPE) should be implemented in the manuscripts to improve the quality of the manuscript. 
v. Results

The outcome of the experiments and study should be depicted in an easy to follow form for better understanding. The sequence of the results should be as per the experimental methods performed. The results should be aligned to the tables, graphs or figures for analysis and verification by the reader. The repetition of data in the tables and figures should be avoided.

Additional information related to the results can be provided as supplementary materials.

vi. Discussion

The discussion should include the analysis of the methods and the results obtained from them. The inference or the significance of the results should be described in detail. This can include content to prove that the improvisation from the previous work cited and its significant outcomes to prove the achievement of the objectives. Caution should be excised not to repeat the information given under introduction and results.

vii. Conclusion

The conclusion should summarise an analytical outcome of the present studies and the results obtained. The significance of the results and discussion should be presented in brief to conclude the study. A scope for further research should be included as a suggestion to continue or steer future research in the present field of study.

viii. Acknowledgments

This section provides you with an opportunity to acknowledge individuals for their contribution and support, extended for the study but are not listed as one of the authors. The support extended in the form of funds or research studies from different institutes or agencies can be attributed here.

ix. References

The references are listed as per the alphabetical order of the first authors in the references. They are numbered using Arabic numerals as 1). The citations in the text should be in brackets as depicted (1). The cited numbered references should be depicted after the full stop or period of the sentence. All the cited references should be listed in the reference section of the manuscript.

The reference of the manuscripts listed should be formatted as per the following examples. 
1. Kumar P, Joe M and Xi BR. Article title. Journal Full Name italics. Year; volume (issue): pg. nos. full. ----- Research article

2. Kumar $P$, Joe $M$ and Xi BR. Book title. Edition. Editor. Publisher. City. Year. ----- Book

3. Research on covid-19. Indian health statistics, India. Weblink, year. - Reference to a Website

Note that all the authors listed until 6 authors of a single reference can be listed as per the aforementioned style.

After the first six authors, the authors to be listed should be depicted by et al. The references in the manuscript can be accepted as long as all the references are consistent with one of the reference styles accepted worldwide.

x. Tables

The tables should be submitted as editable text and not as images. Tables should be placed as a separate document. Number the tables consecutively as per their appearance in the text and place any reference notes below the table body. The table can be named as 'Table 1. "Table name*', the table numbers should be in Arabic numerals and should be cited near the appropriate text.

xi. Artwork

The artwork should be of uniform sizing and lettering for the manuscript. The preferred font styles are Times New Roman, font size 12 and double (2.0) spaced between the lines. Number the illustrations/figures/pictures according to their sequence in the text. Please include all the figures or images with figures legends in a single and separate file numbered in Arabic numerals and should be cited at the appropriate text. The TIFF (or JPG), colour or grayscale photographs (halftones) should be a minimum of 300 dpi. Disproportionately large graphics should not be submitted. Avoid providing files that are optimized for screen use (e.g., GIF, BMP, PICT, WPG) or low-resolution images and graphics that are disproportionately large for the content. Please make sure that artwork files are in an acceptable format [TIFF (or JPEG), EPS (or PDF), or MS Office files] and with the correct resolution.

\section{xii. Supplementary material}

The data in the form of tables, figures and graphs that can be considered for verification by the readers or is further reading for the content in the manuscript are considered supplementary. The supplementary material is submitted as separate documents during the submission that will be available online only.

The Submitted supplementary materials are published exactly as they are received (Excel or PowerPoint files will appear as such online). Referencing of the supplementary material in the manuscript should be similar to other documents, 


\section{Journal of Pharmaceutical Research - Author Guidelines}

except that the title should be sufficed by the word supplementary e.g. supplementary table, supplementary figure, etc. along with numbers in Arabic numerals.

Table 1: Guidelines for manuscript preparation

\section{Guidelines based on types of study}

Consolidated Standards of Reporting Trials (CONSORT)

Standards for Reporting of Diagnostic Accuracy (STARD)

\section{Source web link}

http://www.consort-statement.org/

https://www.equator-

network.org/reporting-guidelines/stard/

https://www.strobe-

statement.org/index.php?id=strobe-home
Strengthening the Reporting of Observational Studies in Epidemiology (STROBE) http://www.prisma-statement.org/
Preferred Reporting Items for Systematic Reviews and Meta-Analyses (PRISMA)
Tables

Figures numbers word count

\begin{tabular}{cccccc}
\hline Research article & $150-250$ & Max 3000 & Max 40 & 3 & 3 \\
\hline Case report & $150-200$ & Max 2000 & Max 20 & 2 & 4 \\
\hline Case series & $150-200$ & Max 2000 & Max 20 & 2 & 4 \\
\hline Review article & Max 300 & Max 8000 & Max 100 & 4 & 6 \\
\hline Mini-review & Max 200 & Max 3000 & Max 30 & 2 & 2 \\
\hline Editorial & NA & Max 1000 & NA & 1 & 1 \\
\hline $\begin{array}{c}\text { Short } \\
\text { Communication }\end{array}$ & Max 150 & Max 1500 & Max 12 & 2 & 1 \\
\hline Commentaries & NA & Max 400 & Max 4 & NA & NA \\
\hline Short notes & NA & Max 300 & Max 2 & NA & NA
\end{tabular}

Note: If the number of tables or figures is less than mentioned in the tables, either tables or figures can be added to compensate for the maximum as mentioned in the table. 


\section{Submission Guidelines}

JPR welcomes author submission of papers concerning using the link www.manuscriptcommunicator.com/JPR

All the manuscripts must be submitted on-line through the journal website 'submit manuscript' link. The Author has to register by filling up all the information in a web form. A new user ID and password will be assigned which can be used to login and submit manuscripts. The author can follow up the manuscripts for publication through the same system.

After the manuscript is readied according to the guidelines provided to the author. The manuscript can be prepared for submission by providing the necessary documents as listed below:

1. Cover letter: This is the letter addressed by the author to the Editor-in-Chief of the journal. The body of the letter may contain the title, type of manuscript, the importance of the study reported in the manuscript and the reason for choosing the journal for publication.

2. Title page: This document should contain the title, running title, authors, their affiliations, email IDs, corresponding author, correspondence address, phone number, the word count of the abstract, word count of the article excluding the abstract and references, number of figures, number of tables and supplementary material.

3. Article file: This should contain the article with abstract, materials and methods, results, discussion, conclusion and references.

4. Tables: All the tables should be with titles and footnotes (if necessary), this document should be provided in editable MS-Word format.

5. Figures: All the figures and images should be numbered (e.g. Figure 1) followed by figure legends.

6. Supplementary material: This document may contain additional parts of a manuscript. They may be in the form of tables, figures and datasets, these may not appear as part of the manuscript and it will be available in the online format.

7. Copyright transfer form: A document for authorization of copyrights transfer.

All these documents may not be required for all types of manuscripts during submission. Read the instructions thoroughly before commencing the submission of the manuscript. We look forward to your association with all your publications in the future.

For any query regarding manuscript/article/document submission, please feel free to contact our journal editorial team by filling the contact form with details of your query. 